\title{
Point-of-Care Sexually Transmitted Infection Diagnostics: Proceedings of the STAR Sexually Transmitted Infection-Clinical Trial Group Programmatic Meeting
}

\author{
Anthony D. Cristillo, PhD, MS, * Claire C. Bristow, PhD, MPH, MSc, $\uparrow$ Rosanna Peeling, PhD, $\neq$ \\ Barbara Van Der Pol, PhD, MPH, \& Sasha Herbst de Cortina, BA, I// Ivan K. Dimov, PhD, ** \\ Nitika Pant Pai, MD, MPH, PhD, †† Dong Jin Shin, BSE,tf Ricky Y.T. Chiu, PhD, $/ / \mid$ \\ Catherine Klapperich, PhD, $\&$ Purnima Madhivanan, MD, MPH, PhD, // \\ Sheldon R. Morris, MD, MPH, † and Jeffrey D. Klausner, MD, MPH
}

\begin{abstract}
The goal of the point-of-care (POC) sexually transmitted infection (STI) Diagnostics meeting was to review the state-of-the-art research and develop recommendations for the use of POC STI diagnostics. Experts from academia, government, nonprofit, and industry discussed POC diagnostics for STIs such as Chlamydia trachomatis, human papillomavirus, Neisseria gonorrhoeae, Trichomonas vaginalis, and Treponema pallidum. Key objectives included a review of current and emerging technologies, clinical and public health benefits, POC STI diagnostics in developing countries, regulatory considerations, and future areas of development. Key points of the meeting are as follows: (i) although some rapid pointof-care tests are affordable, sensitive, specific, easy to perform, and deliverable to those who need them for select sexually transmitted infections, implementation barriers exist at the device, patient, provider, and health system levels; (ii) further investment in research and development of point-of-care tests for sexually transmitted infections is needed, and new technologies can be used to improve diagnostic testing, test uptake,
\end{abstract}

From the *Center for Infectious Diseases Research, Social \& Scientific Systems, Inc, Silver Spring, MD; †University of California San Diego School of Medicine, La Jolla, CA; \$London School of Hygiene and Tropical Medicine, London, UK; §University of Alabama at Birmingham School of Medicine, Birmingham, AL; $\uparrow$ University of California, Los Angeles, CA; ||Florida International University, Miami, FL; **Stanford University, Stanford, CA; † McGill University Health Centre, Montreal, QC, Canada; †JJohns Hopkins University, Baltimore, MD; $\S \S$ Boston University, Boston, MA; and $\uparrow \uparrow$ Public Health Research Institute of India, Mysore, India

Acknowledgments: The STAR STI CTG 2016 Programmatic Meeting for POC STI Diagnostics was held on April 20, 2016 at Social \& Scientific Systems, Inc (SSS) $(8757$ Georgia Avenue, 12th Floor, Silver Spring, MD, 20910). The authors would like to acknowledge attendees of the STAR STI CTG 2016 Programmatic Meeting for POC STI Diagnostics: Lynn Barclay, American Sexual Health Association; Claire C. Bristow, University of California, San Diego; Jose Castro, University of Miami; Ricky Chiu, Phase Diagnostics; Anthony Cristillo, Social \& Scientific Systems, Inc; Carolyn Deal, National Institutes of Health (NIH)/National Institute of Allergy and Infectious Diseases (NIAID)/ Division of Microbiology and Infectious Diseases (DMID); Yvette Delph, SSS; Ivan Dimov, Stanford University; David Ecker, Abbott; Yetunde Fakile, Centers for Disease Control and Prevention; Rick Galli, bioLytical Laboratories; Charlotte Gaydos, Johns Hopkins University; Jonathan Glock, NIH/NIAID/DMID; Thomas Hall, Abbott, Sasha Herbst de Cortina, University of California, Los Angeles; Catherine Klapperich, Boston University; Jeffrey Klausner, University of California, Los Angeles; Carol Langley, Office of the US Global AIDS Coordinator; Jill Long, NIH/NIAID/DMID; Gwendolyn Maddox, SSS; and treatment; (iii) efficient deployment of self-testing in supervised (ie, pharmacies, clinics, and so on) and/or unsupervised (ie, home, offices, and so on) settings could facilitate more screening and diagnosis that will reduce the burden of sexually transmitted infections; (iv) development of novel diagnostic technologies has outpaced the generation of guidance tools and documents issued by regulatory agencies; and (v) questions regarding quality management are emerging including the mechanism by which poor-performing diagnostics are removed from the market and quality assurance of self-testing is ensured.

\section{POC Diagnostics}

Consistent with the National Institutes of Health's definition of point-of-care (POC) diagnostics, ${ }^{1}$ the authors consider POC tests to be those that allow patient diagnoses outside of a typical clinical laboratory. Such tests include those that can be executed in a physician's office, hospital bedside, at the patient's

Purnima Madhivanan, Florida International University; Sean McCarthy, SSS; Sheldon Morris, University of California, San Diego; Philipp Mueller, Hologic; Sara McCurdy Murphy, SSS; Nitika Pant Pai, McGill University; Rosanna Peeling, London School of Hygiene \& Tropical Medicine; Lee Pyne-Mercier, Bill \& Melinda Gates Foundation; Gary Richwald, LA/Orange County HSV/HPV/HELP; Renee Ridzon, Office of the US Global AIDS Coordinator; Greg Scott, Beckman Coulter; DJ Shin, Johns Hopkins University; Joanna Sickler, Roche Molecular Systems, Inc; William Smith, National Coalition of STD Directors; Bradley Stoner, Washington University in St. Louis; Delmyra Turpin, NIH/NIAID/DMID; Barbara Van Der Pol, University of Alabama at Birmingham; Toni Waymer, SSS; Peter Wolff, NIH/ NIAID/DMID; and John Waldeisen, Diassess Inc. The STAR STI CTG programmatic meeting was supported by the NIAID contract HHSN2722013000141.

Conflict of interest: None declared.

Correspondence: Anthony D. Cristillo, PhD, MS, Social and Scientific Systems, Inc, Silver Spring, MA. E-mail: acristillo@s-3.com.

Received for publication October 10, 2016, and accepted November 21, 2016.

\section{DOI: $10.1097 /$ OLQ.0000000000000572}

Copyright $\odot 2017$ The Author(s). Published by Wolters Kluwer Health, Inc. on behalf of the American Sexually Transmitted Diseases Association. This is an open-access article distributed under the terms of the Creative Commons Attribution-Non Commercial-No Derivatives License 4.0 (CCBY-NC-ND), where it is permissible to download and share the work provided it is properly cited. The work cannot be changed in any way or used commercially without permission from the journal. 
home, or in the field. Point-of-care testing facilitates the provision of rapid treatment and care to the patient. Some of the desired attributes of POC sexually transmitted infection (STI) tests include (i) accuracy ( $>95 \%$ ), (ii) ease of use/minimal training required, (iii) rapid turnaround time (while the patient is still accessible), (iv) low cost, and (v) portability. Historically, there have been rapid POC tests to aid in sexually transmitted disease diagnosis such as the Gram stain (Neisseria gonorrhoeae [NG]), rapid plasma reagin (syphilis), POC microscopy (trichomoniasis), vaginal whiff (bacterial vaginosis [BV]), $\mathrm{pH}$ testing, and POC microscopy with and without potassium hydroxide for vaginal candidiasis and BV. Those tests are widely used in a variety of clinical settings, and although they can be considered POC tests, they were outside the scope of our discussion.

\section{The Need for POC STI Diagnostics}

Chlamydia trachomatis (CT), human papillomavirus (HPV), N. gonorrhoeae, Trichomonas vaginalis (TV), and Treponema pallidum (TP), among the most common causes of STIs, contributed approximately 357 million new STI cases globally in 2012 . In 2012, there were 131 million cases of CT, 78 million cases of NG, 143 million cases of TV, and 5.6 million cases of syphilis, caused by T. pallidum. ${ }^{2}$ In addition, more than 290 million women have an HPV infection, which leads to cervical cancer. ${ }^{2}$ Early and accurate diagnosis of infection is important to provide patients with timely, appropriate, and effective treatment, thereby, limiting the spread and adverse outcomes of infection. Approximately $80 \%$ of the newly reported STI cases occur in developing countries, and they disproportionately impact women. ${ }^{3}$ Many STI patients are asymptomatic and may be identified only through screening. ${ }^{4}$ Without accurate tests, these asymptomatic cases could remain unknowingly chronically infected and untreated for an extended period of time, resulting in long-term reproductive complications and further spread of infections to others.

Consistent with the 2030 Agenda for Sustainable Development, the World Health Organization (WHO) has developed draft global health sector guidance on STIs. ${ }^{5}$ The three draft strategies included (i) universal health coverage, (ii) the continuum of health services, and (iii) the public health approach. Each strategy was established with a vision, a goal, targets, and actions towards eliminating STIs as public health threats, and actions to be taken by countries, WHO, and partners. Point-of-care diagnostics can facilitate achievement of the vision set forth for STIs in those sustainable development goals.

\section{Reproductive and Maternal Health}

Although common among pregnant women, STIs such as CT and NG may lead to adverse birth outcomes. ${ }^{6,7}$ Syphilis, CT, $\mathrm{NG}$, and TV in pregnancy can cause fetal death, stillbirth, low birth weight, preterm birth, fetal growth retardation, neonatal death, and infections in infants. ${ }^{6,8-11}$ STI screening and treatment in pregnancy can prevent such adverse outcomes. ${ }^{8,12-14}$ Detection and treatment of CT during pregnancy have been shown to reduce ocular disease in newborns, and treatment of NG during pregnancy reduces pregnancy-related complications. ${ }^{15,16}$ Challenges still remain in the uptake of screening, the availability of diagnostics, and screening policies for antenatal care in developing countries. For example, a 2015 Progress Report for the Implementation of the Global Strategy for Prevention and Control of Sexually Transmitted Infections: 2006-2015 reported that the median percentage of antenatal care attendees who were tested for syphilis remained largely unchanged from $2008(59 \%)$ to $2013(58 \%)$ in the WHO African region. ${ }^{17}$ Furthermore, in the WHO Eastern Mediterranean region, many countries had no syphilis diagnostic tests or screening policies for pregnant women because of a perceived low incidence. ${ }^{17}$ However, syphilis screening in antenatal care has been shown to be cost-effective even in low prevalence settings. ${ }^{18}$

\section{Lack of Access, Equipment, and Trained Personnel}

Although STI diagnostic tests are available and used in high-income countries, they are largely unavailable in low- to middle-income countries where there is a lack of equipment and trained personnel required for etiological diagnosis of STIs. ${ }^{2,19,20}$ Additional challenges associated with current diagnostics include high cost of available tests and delayed turnaround time for patients to receive results. However, POC STI diagnostics that are available for syphilis are accurate, easy to use, and provide rapid results. ${ }^{21}$ Hence, extending the reach of such POC diagnostics would have a significant impact on early STI diagnosis and treatment if implemented in national, regional or district laboratories, primary care facilities and clinics, bedside, nonfacility-based community outreach programs, and within homes. The success of POC syphilis screening underscores the necessity to deliver POC screening to all major STIs.

\section{Etiological Versus Syndromic Management}

To date, STI etiological diagnosis and management has been challenging. Although standard nucleic acid amplification instruments have become smaller and methods are near real time, such tests can be technically challenging and require expensive equipment and skilled laboratory or medical staff. Such resources are not easily available in low- to middle-income countries. Hence, syndromic management continues to be the primary approach recommended by public health professionals for STI treatment $17,22,23$ STI syndromic management is based on the identification of several easily recognized signs and symptoms. However, this approach lacks specificity and symptoms may reflect nonsexually transmitted conditions. ${ }^{24}$ That approach could lead to inappropriate overtreatment, resulting in antimicrobial resistance, continued transmission, and unnecessary drug toxicity. Syndromic management also does not address asymptomatic infections, which are of public health concern. Given its low specificity and sensitivity, syndromic management is unlikely to impact the burden of disease. By contrast, the development of highly specific and sensitive POC STI diagnostics could facilitate a departure from syndromic management in favor of etiological management of STIs. Such diagnostics could facilitate appropriately targeted treatments because of rapid specific STI detection, sparing unnecessary use of antimicrobials that may contribute to resistance. By implementing a POC diagnostics screening program, progress would also be made to identify infected asymptomatic patients.

\section{POC STI Diagnostics and Unique Challenges/Considerations for Developing Countries}

A lack of access to medical facilities and affordable testing are likely reasons why STI services fail. In countries such as Benin, Bolivia, Chad, Haiti, Madagascar, Niger, Tanzania, Uganda, and Zimbabwe, patients must often travel $4.7 \mathrm{~km}$ or more to access the nearest medical facility. ${ }^{25}$ Relative inaccessibility of medical facilities raises the concern that, if treatment is not provided during a diagnostic visit, loss to follow-up is highly likely. Thus, even in countries where diagnostic testing is available, limitations of accessibility restrict the number of STI patients who can benefit from early diagnosis and treatment. 
While trying to satisfy the overall goals of quality, timeliness, reliability, and cost, one needs to be aware of tradeoffs between performance and cost when assessing POC STI diagnostics for use in resource-limited settings. Experts within the programmatic meeting cited the ASSURED ( $\mathrm{A}=$ affordable; $\mathrm{S}=$ sensitive; $\mathrm{S}=$ specific; $\mathrm{U}=$ user-friendly; $\mathrm{R}=$ rapid and robust; $\mathrm{E}=$ equipment free; $\mathrm{D}=$ deliverable) criteria as a useful approach to evaluate POC STI diagnostics. ${ }^{26}$ In more urban settings, accuracy could be considered a more important factor as compared with cost or rapid turnaround time for results. ${ }^{26,27}$ By contrast, in more rural settings, cost and the delivery of rapid results could outweigh accuracy. To that end, a less sensitive but more accessible test may be more easily deployed and impactful in places where individuals may not currently have access to any STI diagnostics.

\section{CURRENT STATE OF POC STI DIAGNOSTICS}

A number of POC STI diagnostic platforms are available and provide results within 25 to 90 minutes; however, their performance is quite variable. ${ }^{28-39}$ Diagnostic platforms include antibody and antigen detection assays as well as nucleic acid amplification tests (NAATs). Such tests include immuno-chromatographic tests such as ACON Chlamydia and ACON NG/CT tests (ACON Laboratories, San Diego, Calif); BioRapid Chlamydia Ag Test (Biokit S.A., Barcelona, Spain); Chlamydia Rapid Test SAS (Diagnostics for Real World, Cambridge, UK); Clearview Chlamydia (Alere, Waltham, Md); Chlamydia test card (Ultimed Products, GmbH, Germany); Ultimed NG Test and Chlamydia Ag Test Card (Ahrensburg, Germany); QuickVue Chlamydia Rapid Test (Quidel Corporation, San Diego, Calif); and real-time polymerase chain reaction (eg, Xpert CT/NG, Cepheid, Sunnyvale, Calif) assays. Additional POC STI testing platforms include io CT, io CT/NG, and io TV (Atlas Genetics Ltd, Trowbridge, UK), Trulab PCR CT/NG, (Molbio/bigTec, Goa, India), Alere-I/Alere (CT/NG), ${ }^{28}$ and the colorimetric antigen-targeting OSOM rapid TV test (Sekisui Diagnostics, LLC, Lexington, Md). Although sensitive and specific POC tests are available for $\mathrm{CT}, \mathrm{NG}$, and TV, progress is needed to improve availability and performance of some currently available diagnostic tests.

\section{POC Syphilis and HIV/Syphilis Dual Tests}

There are several rapid POC tests for syphilis. These tests are antibody detection lateral flow assays and include the Alere Determine Syphilis TP, the SD BIOLINE Syphilis 3.0 test, and the Chembio DPP Syphilis Screen \& Confirm Assay. For whole blood syphilis tests, sensitivity can range from $86.3 \%$ to $100 \%$ and specificity, from $95.9 \%$ to $97.0 \%{ }^{40}$

Human immunodeficiency virus (HIV) and syphilis coinfection is increasingly common, and it has been found that syphilis infection may facilitate HIV acquisition and transmission including mother-to-child transmission of HIV. ${ }^{4-45}$ Dual POC rapid tests detect antibodies to both HIV and T. palladium and have the potential to simplify training, streamline procurement, ensure testing for both HIV and syphilis, and improve patient experiences. The similarities in screening recommendations for HIV and syphilis offer an important opportunity to strengthen prevention programs for the elimination of congenital syphilis along with prevention of HIV mother-to-child transmission by means of integrated screening. ${ }^{30}$ Dual rapid HIV/syphilis tests make integrated screening more feasible, and there are currently at least 5 manufacturers of these tests (Chembio Diagnostics Systems Inc, Medford, NY; MedMira Inc, Halifax, Canada; Standard Diagnostics Inc, Gyeonggi-do, Republic of Korea; Premier Medical Corp Ltd, Watchung, NJ; bioLytical Laboratories, Inc, British Columbia,
Canada) and 1 dual assay prequalified by WHO (Alere SD Bioline HIV/Syphilis Duo Test).

Dual HIV/syphilis rapid tests use specific recombinant antigens, which form an antigen-antibody complex when specific HIV and/or syphilis antibodies are present in the specimen. Such POC tests use finger stick whole blood sample and a single device to test for both HIV and syphilis in minutes. Evaluations of dual $\mathrm{HIV} /$ syphilis tests revealed that HIV sensitivity can range from $95 \%$ to $100 \%$ in the laboratory ${ }^{30-36}$ and $91 \%$ to $99 \%$ in field settings. ${ }^{37,38}$ Human immunodeficiency virus specificity can range from $94 \%$ to $100 \%$ in the laboratory and $97 \%$ to $100 \%$ in the field. ${ }^{37,39}$ Treponemal sensitivity in the same dual HIV/syphilis tests can range from $87 \%$ to $100 \%$ in the laboratory and $80 \%$ to $96 \%$ in the field; treponemal specificity can range from $93 \%$ to $100 \%$ in the laboratory and $91 \%$ to $100 \%$ in the field. Relative to single testing strategies, dual HIV/syphilis tests demonstrated good performance, fewer finger pricks and/or blood draws, increased efficiencies in service delivery (eg, time saving, streamlined procurement, ensured testing for 2 infections for every patient), and greater cost-effectiveness. ${ }^{46}$ Treponemal antibodies can persist for life; therefore, in settings where there is high prevalence of previously treated syphilis, such as in men who have sex with men (MSM) populations, confirmatory tests may be needed before treatment initiation. In some settings, such as in resourcelimited areas and in antenatal care, the risk of untreated infection may outweigh the risk of unnecessary treatment. In addition to dual tests for HIV and syphilis, there is a need in some settings, in particular in Asia where hepatitis B is endemic, for triplex assays to detect HIV, syphilis, and hepatitis B infection. ${ }^{47}$ The evaluation of multiplex testing algorithms in antenatal care settings is urgently needed. ${ }^{48}$

\section{POC tests for CT, NG, and TV}

Among current POC tests for CT, NG, and TV, Cepheid's NG/CT GeneXpert assay uses multiplex, rapid real-time polymerase chain reaction and fluorescence detection. Point-of-care testing for those STIs can also be accomplished by lateral flow immunoassays (LFAs) such as the aQcare Chlamydia TRF test (Medisensor Inc, Seoul, Korea), QuickVue CT, and QuickVue TV test (Quidel Corporation, San Diego, Calif). ${ }^{28,29}$ For all POC CT tests, sensitivity can range from $17.1 \%$ to $98.7 \%$, whereas specificity can range from $91.3 \%$ to $100 \%{ }^{28}$ In a literature review of NG and TV tests, it was found that sensitivity can range from $12.5 \%$ to $100 \%{ }^{49,50}$ and $38 \%$ to $98 \%,{ }^{51-53}$ respectively, whereas specificity can range from $93 \%$ to $100 \%{ }^{50,54,55}$ and $99.3 \%$ to $100 \%,{ }^{51,56}$ respectively. ${ }^{28}$ Although there are sensitive and specific tests for CT (GeneXpert, aQcare Chlamydia TRF), NG (GeneXpert), and TV (OSOM), the implication of the wide sensitivity ranges across different tests is missed infections and continued transmission to partners and unborn children.

\section{NEW STI POC DIAGNOSTICS AND APPROACHES}

\section{Enhanced CT Lateral Flow Assay}

Honesti (Phase Diagnostics, Santa Ana, Calif) is a new, simple, rapid, handheld POC diagnostic for the detection of CT infection. ${ }^{57}$ Although the device is based on the conventional LFA, it additionally incorporates a novel target concentration mechanism to overcome the sensitivity limitations of other lateral flow tests for CT. Concentration of the target is accomplished by using a proprietary aqueous two-phase system that enriches the target biomarker before detection, significantly improving the detection limit of conventional LFAs. These aqueous two-phase system components are inexpensive, require no additional power source, 
and are integrated such that no additional training is required. From the end user's point of view, the device is performed no different from the conventional LFA (eg, pregnancy test), allowing for self-sample collection and colorimetric readout in less than 10 minutes. Proof-of-concept studies have been conducted on clinical CT samples, bacteria, viruses, proteins, and small molecules. ${ }^{57-59}$ Given its unique targeted concentration mechanism, Honesti could provide detection of $\mathrm{CT}$ in patient samples with low CT concentrations; however, clinical trials are urgently needed to validate this. This simple platform has the potential to increase screening access and enable true same visit testing and treatment in decentralized settings.

\section{Cell Phone-Based DNA CT Test}

In conventional lateral flow processing, fluids move through the system, whereas the analyte(s) stays in place. A droplet magnetofluidic process has been developed, whereby the fluid stays in place and the analyte moves through the system. ${ }^{60,61}$ This principle uses reagents in the form of aqueous drops, sealed in immiscible oil. The structural/surface energy-based trapping facilitates droplet isolation, and hydrophobic surfaces facilitate transport of magnetic beads on planar surfaces. Potential benefits of this new fluid processing are that it is compatible with reagents and assays as well as that its simple design is amenable to use with low-cost instrumentation. MobiNAAT, a cell phone-based DNA CT test, integrates a mobile telephone and a cartridge, which serves as the processing unit for a loop-mediated isothermal amplification assay. The cartridge is responsible for (i) thermal incubation, (ii) magnetic particle handling, and (iii) optical path imaging. The cartridge facilitates sample processing coordination and optical signal preprocessing. A mobile phone provides the user interface (UI), video tutorials, and data acquisition/signal processing through the phone's camera. The user can use a mobile application to assist with platform workflow. In a retrospective study of vaginal swabs for CT, 20 archived dry vaginal swabs (10 positive and 10 negative) were tested using the MobiNAAT technology. Investigators at Johns Hopkins University found that results were in full agreement with the reference results (unpublished data). An on-site evaluation of the test at the Johns Hopkins University Emergency Department found that MobiNAAT could produce CT test results in approximately 1 hour, whereas central laboratory testing required 80 hours to yield comparable results (unpublished data). In addition, the costs associated with that test are much lower than those of standard POC NAATs. Given that MobiNAAT uses a smartphone and small cartridge, it requires significantly less space than a typical POC NAAT. In resourcelimited settings, where there is a lack of infrastructure to implement POC diagnostics that require specialized training, expensive equipment, and/or laboratory infrastructure, the widespread availability of smartphones could make MobiLab tests a feasible means to facilitate POC diagnostic testing.

\section{Other Key Innovations}

To make molecular diagnostics more readily available in developing countries, disposable molecular diagnostic systems that can be used via a smartphone are currently being developed. Three key areas of innovations include (i) self-powered microfluidic flow, (ii) robust sample preparation, and (iii) diagnostic assays with sensitive visual readouts. To those ends, integrated universal sample preparation modules are being developed using microsedimentation-based cell separation, reagent-free electrochemical cell lysis on a chip, and pumpless degas flow. Other innovations include miniaturization using Nanowire/Nanodot technologies, which offer sensitive pathogen detection with minimal sample collection. Nanowire technologies have been used to detect malaria parasites, distinguish different species, and identify drug resistance using a finger-pricked sample of blood within 20 minutes.

\section{Paper Fluidic Diagnostics or Paper-Plastic Hybrid Molecular Diagnostics for CT/NG}

Over the past 5 years, efforts to improve molecular diagnostics for CT and NG have not only focused on microfluidic diagnostics but have used paper fluidic or paper-plastic hybrid molecular diagnostics. Such molecular tests have targeted human papillomavirus and tried to produce same day results that could be read without instrumentation. To accomplish these goals, Klapperich and coworkers have developed a method of extracting nucleic acids into a planar platform/two-dimensional layout and have applied this technology to extract nucleic acid from human samples. ${ }^{62}$ Once a swab is collected, it is placed in a buffer that facilitates nucleic acid extraction onto a membrane. Once extracted, nucleic acids are amplified. For that, a number of isothermal amplification techniques including helicase dependent amplification have been incorporated onto the planar platform. Standard LFA technology allows this test to produce a nonquantitative, "positive/negative" result that is visible on paper. Klapperich and coworkers are also developing a similar test for $\mathrm{NG}$ and are working toward a multiplexing assay to detect both CT and NG. Currently, to obtain results, the user must perform sample collection, washing, and elution. Ongoing efforts are aimed at reducing the number of steps involved and to reduce the overall turnaround time from less than 1 hour to 15 minutes.

\section{Simple Steps: Optimizing Diagnostic Strategies by Adding Vaginal $\mathrm{pH}$ to Testing Algorithms}

In settings where new diagnostic tests could take several years to successfully implement, existing tools such as $\mathrm{pH}$ testing can be incorporated to enhance syndromic management. The $\mathrm{pH}$ of the upper vagina is normally acidic ( $\mathrm{pH}$ level of 3.8 to 4.5), whereas bacterial overgrowth in $\mathrm{BV}$ and trichomonas can increase vaginal $\mathrm{pH}$ higher than 4.5. Elevated vaginal $\mathrm{pH}$ can therefore serve as a marker for $\mathrm{BV}$ or $\mathrm{TV}$ infection. A study conducted in India, in which 898 sexually active adult women were screened for reproductive tract infections, found that vaginal $\mathrm{pH}$ and whiff test together correctly diagnosed $82 \%$ of laboratorydiagnosed BV cases versus $40 \%$ with syndromic management and $83 \%$ of laboratory-diagnosed TV infections versus $51 \%$ with syndromic management. $^{63}$

Clarke and coworkers found that elevated vaginal $\mathrm{pH}$ was associated with greater risk of infection with multiple HPV types in women younger than 35 years and older than 65 years. ${ }^{64}$ Therefore, in settings where visual inspection is used in lieu of Pap smears, the addition of vaginal $\mathrm{pH}$ could potentially assist in the detection of HPV in women within these age groups. Additional studies in Israel and Africa have shown that vaginal $\mathrm{pH}$ testing could improve the diagnosis of vaginal candidiasis. ${ }^{65}$ As such, an algorithm combining vaginal $\mathrm{pH}$ testing and subjective evaluation of discharge could have a higher sensitivity and specificity than the WHO syndromic management testing strategy.

\section{POC Diagnostics and New Approaches to Self-Testing}

Problems associated with conventional testing include social visibility, a lack of confidentiality, and long turnaround times for results and treatment, as well as stigma and discrimination. Such problems are not restricted to developing countries but reflect global issues associated with conventional testing. Self- 
testing could mitigate those issues by providing confidential and convenient testing that is personalized and affordable. Selftesting could provide links to care with a shorter turnaround time than conventional testing. Two types of self-test strategies are: (i) unsupervised and (ii) facilitated/supervised self-testing. In unsupervised self-testing, users understand the pretest information, conduct and interpret the self-test, and follow through on results themselves. In facilitated testing, the self-testing is conducted by the participant with the aid of counselors in a supervised setting. ${ }^{66}$ A unique form of facilitated testing through self-collection is available in settings such as the Dean Street Express clinic in London's SoHo district. In that site, asymptomatic persons can (i) register, receive risk-based screening recommendations, and obtain specimen labels via touch screen computers; (ii) perform their own specimen collection in clinic bathrooms; (iii) submit specimens for testing (Cepheid's GeneXpert CT/NG) via a pressurized air tube system; and (iv) obtain texted results via short message service for CT or NG in less than 6 hours. Such unique approaches to leverage technology to facilitate confidential selftesting have proven successful in reducing the time to treatment. ${ }^{67}$ Although self-collection and the use of Xpert tests may be appropriate in resource-rich countries, their implementation in resourcepoor nations may be more challenging. Although self-testing for HIV is being widely promoted and supported by funders, national control programs are encountering issues with regulatory approval and linkage to care. ${ }^{68}$ Progress made in resolving these challenges will facilitate self-testing for STIs.

\section{GENERAL POC CHALLENGES}

Given that there are POC tests with widely varying sensitivities, ${ }^{28}$ countries are challenged to identify and select the best platforms for their sites. New accurate technologies for POC STI tests, especially for use during pregnancy, are urgently needed. Costeffectiveness studies could serve as a tool to help policy makers identify the interventions, which maximize health resources available in their setting and improve resource allocation and management activities. In public health settings, choices must be made about which health strategies to use; a cost-effectiveness analysis can provide evidence about how to prioritize resources.

\section{IMPLEMENTATION BARRIERS}

Although POC testing can significantly impact global health, barriers to implementation exist at the device, patient, provider, and health system levels. ${ }^{69}$ At the device level, barriers include a lack of assay robustness and accuracy, a lack of integration of multiple tests targeting different pathogens, and difficulties in performing and interpreting the POC test. At the patient level, barriers include costs for confirmatory tests, difficulties in performing the POC test, distrust, lack of awareness, privacy issues, and/or a preference for conventional approaches. ${ }^{69}$ At the provider level, barriers include costs, a lack of awareness, complicated protocols, challenges in workflow, or simply a resistance to adopting POC diagnostics. At the health system level, barriers include costs, integration challenges, quality assurance and quality control requirements, workflow challenges, required training, and technical support. ${ }^{69}$ Among the workflow challenges that could arise is the reporting of notifiable STIs. In the United States, such reporting is currently performed by clinical laboratories.

\section{REGULATORY CHALLENGES OF NEW TECHNOLOGY POCS}

To meet global public health needs, new technologies that facilitate development of POC STI diagnostics have emerged. Although some of those tests are focused on single pathogen detection, others leverage multiplexing technologies. However, development of new technologies has outpaced the generation of guidance tools and documents by regulatory agencies. Although some guidance documents exist for POC STI diagnostics, those recommendations are based on older technology. ${ }^{70}$ Different technologies yield different results, and performance (eg, sensitivity) is dependent on the technology deployed. Questions therefore remain as to what constitutes acceptable performance given the tension between where a POC diagnostic is needed and how it will be used. Therefore, factors such as accuracy and access must be considered when deciding how multiplex devices will be evaluated. ${ }^{48,71}$ The current environment may also not be ideal to newly developed assays. As the field continues to move forward at a rapid pace, questions are emerging regarding quality management. One question, for example, is who would be responsible for maintaining and calibrating controls as well as deciding the fate for existing STI tests that are not performing well or can be replaced with newer platforms. ${ }^{72}$ Other issues that will require clarification and guidance include difficulties with limits of detection and with inclusivity experiments. Verification of off-label use for existing POC STI tests will also require some inputs from regulatory agencies.

Many POC assays are available outside the United States, and although some have reasonable diagnostic performance, the majority of tests do not. This is especially true for developing countries where cost is of paramount importance. From a public health perspective, it may be beneficial to have many assays available because they can lower market costs, expand the reach beyond routine clinical settings, and maximize resources by performing multiplex testing. Partnerships between academia, industry, and the Food and Drug Administration (FDA) may help facilitate development of more adaptable guidance systems. The goals of such a partnership should be fair to all manufacturers and an affordable path to market for high quality tests. The field could also benefit from academic and industry stakeholders providing support to the FDA to find ways to work within the regulatory environment while still meeting public health needs.

\section{RESEARCH PRIORITIES, CONCLUSIONS, AND FUTURE DIRECTION}

\section{Clinical Research Priorities}

With regard to syphilis, key clinical research priorities include (i) antenatal TP screening and its impact on morbidity and (ii) identifying syphilis infections in MSM requiring treatment. Given that congenital syphilis continues to be of public health concern with nearly 1 million pregnant women infected with syphilis each year, a study of POC syphilis testing designed to demonstrate improvements of birth outcomes in pregnant women who are screened and treated for syphilis is needed. ${ }^{73}$ For studies directed at treating MSM, incorporating a POC syphilis/HIV dual test could be effective at early detection. However, as mentioned above, for MSM populations, confirmatory tests may be needed before treatment initiation given the persisting treponemal antibodies. Hence, replacing blood tests for syphilis with multiplexing POC diagnostic tests could facilitate earlier diagnosis of STIs. Other antenatal screening priorities identified include tests for $\mathrm{CT}$ and $\mathrm{NG}$ in pregnant women and their infants.

Other priorities discussed include the need to implement POC diagnostic testing in primary care settings. As discussed above (Implementation Barriers, Regulatory Challenges of New Technology POCs), clinical and systemic attitudes towards POC testing are generally not good due to POC implementation hurdles and regulatory challenges. The question was raised whether an existing screening approach, modeled on rapid POC influenza 
testing, could be used to implement POC STI testing. The working group proposed the use of POC STI testing in urgent care settings where patients expect real-time clinical management. As discussed, STI etiological diagnosis and management has been challenging, especially in resource-limited settings; hence, syndromic management continues to be the primary approach recommended by public health professionals for STI treatment. ${ }^{17,22,23}$ As a result, many providers are not incentivized to find better methods for testing and treatment. As noted above, syndromic management is ineffective, leads to antimicrobial overuse, and likely furthers resistance.

\section{Laboratory Research Priorities}

One of the key questions identified was whether the use of POC diagnostic tests with lower sensitivity is acceptable for use. Such tests may be acceptable for use in certain settings where patients are not routinely tested or treated for STIs. In other words, there is a need to balance the sensitivity of POC tests with overall access to care.

In addition to an expansion of existing tests that perform well, exciting new methods of pathogen detection were discussed. The ability to miniaturize tests, multiplexing technologies, and paper-based detection methods to reduce costs are all promising approaches. In regards to nanotechnology, use of nanochords or particles to amplify signals is promising development for POC STI diagnostics. Nanotechnology could therefore be used to make the detection of STIs better, faster, and easier.

\section{Policy Priorities}

A barrier to POC test development is how the current regulatory environment often makes it difficult to bring dual and multiplex diagnostic tests into practice. An additional challenge is in the regulation of multiplexed tests, where FDA requirements for analytical and clinical performance may be too stringent and insurmountable. It was therefore suggested that public/private partnerships could help inform changes to the FDA regulation of multiplexing tests.

With respect to costs for POC diagnostics, the ideal price point identified was about US $\$ 1.00$ per test in low-resource settings. Although making tests too inexpensive could deincentivize production, higher costs could limit adoption of such tests in developing countries. Consensus was that, in the United States, an average cost of US $\$ 20.00$ for diagnostics tests would be reasonable.

In summary, although there is a clear and urgent need for rapid POC diagnostics for STIs, the field is in its very early stages. There are exciting new developments in diagnostic technology, but limited demand and challenges in the regulatory approval of such devices exist. Funders and developers will need vision and steady motivation to bring new diagnostics forward. There is great potential for public health benefit with the advent of POC STI diagnostics. When and how that potential will be realized, however, remains to be seen.

\section{REFERENCES}

1. National Institutes of Health. Point-of-Care Diagnostic Testing Fact Sheet. 2010. Available at: https://report.nih.gov/nihfactsheets/ ViewFactSheet.aspx?csid=112.

2. World Health Organization. Sexually Transmitted Infections: Fact Sheet $\mathrm{N}^{\circ} 110.2015$. Available at: http://www.who.int/mediacentre/ factsheets/fs110/en/

3. CDC and World Bank. 2008. Sexually Transmitted Infections in Developing Countries. Current Concepts and Strategies on Improving STI Prevention, Treatment, and Control. Available at: http://siteresources. worldbank.org/INTPRH/Resources/STINoteFINAL26Feb08.pdf.
4. Tomnay JE, Coelli L, Hocking JS. High rates of chlamydia found among 12- to 16-year-olds attending a rural sexual health clinic: Implications for practice. Sex Health 2016; 13:193-195. doi: 10.1071/ SH15140.

5. World Health Organization 69th World Health Assembly. 2016-2021 Global Health Sector Strategies for HIV, Viral Hepatitis, and Sexually Transmitted Infections. Available at: http://www.who.int/hiv/strategy2016-2021/Online_Consultation_Report_16092015.pdf?ua=1.

6. Adachi K, Klausner JD, Xu J, et al. Chlamydia trachomatis and Neisseria gonorrhoeae in HIV-infected Pregnant Women and Adverse Infant Outcomes. Pediatr Infect Dis J 2016; 35:894-900. doi: 10.1097/ INF.0000000000001199.

7. LeFevre ML. Screening for chlamydia and gonorrhea: U.S. Preventive Services Task Force recommendation statement. Ann Intern Med 2014; 161:902-910. http://doi.org/10.7326/M14-1981.

8. Gomez GB, Kamb ML, Newman LM, et al. Untreated maternal syphilis and adverse outcomes of pregnancy: A systematic review and meta-analysis. Bull World Health Organ 2013; 91:217-226. http:// doi.org/10.2471/BLT.12.107623.

9. Johnston VJ, Mabey DC. Global epidemiology and control of Trichomonas vaginalis. Curr Opin Infect Dis 2008; 21:56-64. http://doi.org/ 10.1097/QCO.0b013e3282f3d999.

10. Romoren M, Hussein F, Steen TW, et al. Costs and health consequences of chlamydia management strategies among pregnant women in sub-Saharan Africa. Sex Transm Infect 2007; 83:558-566. http:// doi.org/10.1136/sti.2007.026930.

11. Su WH, Tsou TS, Chen CS, et al. Are we satisfied with the tools for the diagnosis of gonococcal infection in females? J Chin Med Assoc 2011; 74:430-434. http://doi.org/10.1016/j.jcma.2011.08.012.

12. Braccio S, Sharland M, Ladhani SN. Prevention and treatment of mother-to-child transmission of syphilis. Curr Opin Infect Dis 2016; 29:268-274. http://doi.org/10.1097/QCO.0000000000000270.

13. Chen XS, Yin YP, Chen LP, et al. Sexually transmitted infections among pregnant women attending an antenatal clinic in Fuzhou, China. Sex Transm Dis 2006; 33:296-301. http://doi.org/10.1097/01. olq.0000194580.02116.6b.

14. Kuznik A, Lamorde M, Nyabigambo A, et al. Antenatal syphilis screening using point-of-care testing in Sub-Saharan African countries: A cost-effectiveness analysis. PLoS Med 2013; 10:e1001545 http://doi.org/10.1371/journal.pmed.1001545.

15. Hammerschlag MR. Chlamydial and gonococcal infections in infants and children. Clinical Infectious Diseases. 2011; 53:S99-S102. http://doi.org/10.1093/cid/cir699.

16. Moore DL, MacDonald NE. Preventing ophthalmia neonatorum. Can J Infect Dis Med Microbiol 2015; 26:122-125. http://doi.org/10. $1155 / 2015 / 720726$

17. World Health Organization (2015). Implementation of the Global Strategy for Prevention and Control of Sexually Transmitted Infections: 2006-2015 (Progress Report). Available at: http://apps.who.int/ iris/bitstream/10665/183117/1/9789241508841_eng.pdf?ua $=1$.

18. Mallma P, Garcia P, Carcamo C, et al. Rapid syphilis testing is costeffective even in low-prevalence settings: The CISNE-PERU experience. PLoS One 2016; 11:e0149568. http://doi.org/10.1371/journal. pone. 0149568

19. Tucker JD, Bien CH, Peeling RW. Point-of-care testing for sexually transmitted infections: Recent advances and implications for disease control. Curr Opin Infect Dis 2013; 26:73-79. http://doi.org/10.1097/ QCO.0b013e32835c21b0.Point-of-care.

20. World Health Organization. (2013). Sexually Transmitted Infections (STIs). The Importance of a Renewed Commitment to STI Prevention and Control in Achieving Sexual and Reproductive Health. Available at: http://doi.org/10.1111/jmwh.12094.

21. Jafari Y, Peeling RW, Shivkumar S, et al. Are Treponema pallidum specific rapid and point-of-care tests for syphilis accurate enough for screening in resource limited settings? Evidence from a meta-analysis. PLoS One 2013; 8:e54695. doi: 10.1371/journal.pone.0054695. Epub 2013 Feb 26.

22. World Health Organization. Report on Global Sexually Transmitted Infection Surveillance 2013. 2014. Available at: http://apps.who.int/iris/ bitstream/10665/112922/1/9789241507400_eng.pdf?ua=1. 
23. World Health Organization. Global Incidence and Prevalence of Selected Curable Sexually Transmitted Infections - 2008. (2012). Available at: http://doi.org/10.1016/S0968-8080(12)40660-7.

24. Garrett NJ, McGrath N, Mindel A. Advancing STI care in low/middleincome countries: Has STI syndromic management reached its use-by date? Sex Transm Infect 2016. pii: sextrans-2016-052581. doi: 10.1136/ sextrans-2016-052581.

25. World Health Organization. 2004. The World Health Report 2004: Changing History. Available at: http:/www.who.int/whr/2004/en/report04_en.pdf?ua=1.

26. Peeling RW. Diagnostics in a digital age: An opportunity to strengthen health systems and improve health outcomes. Int Health 2015; 7 : 384-389. doi: 10.1093/inthealth/ihv062.

27. Okeke IN, Peeling RW, Goossens H, et al. Diagnostics as essential tools for containing antibacterial resistance. Drug Resist Updat 2011; 14:95-106. http://doi.org/10.1016/j.drup.2011.02.002.

28. Herbst de Cortina S, Bristow CC, Joseph Davey D, et al. A Systematic Review of Point of Care Testing for Chlamydia trachomatis, Neisseria gonorrhoeae, and Trichomonas vaginalis. Infect Dis Obstet Gynecol 2016:4386127. http://doi.org/10.1155/2016/4386127.

29. Posthuma-Trumpie GA, Korf J, van Amerongen A. Lateral flow (immuno)assay: Its strengths, weaknesses, opportunities and threats. A literature survey. Anal Bioanal Chem 2009; 393:569-582. doi: 10.1007/s00216-008-2287-2.

30. Bristow CC, Klausner JD. Cuba: Defeating mother-to-child transmission of syphilis. Lancet 2015; 386:1533. doi: 10.1016/S0140-6736 (15)00485-7.

31. Bristow CC, Adu-Sarkodie Y, Ondondo RO, et al. Multisite laboratory evaluation of a dual human immunodeficiency virus (HIV)/syphilis point-of-care rapid test for simultaneous detection of HIV and syphilis infection. Open Forum Infect Dis 2014; 1:ofu015. doi: 10.1093/ofid/ ofu015. eCollection 2014.

32. Humphries RM, Woo JS, Chung JH, et al. Laboratory evaluation of three rapid diagnostic tests for dual detection of HIV and Treponema pallidum antibodies. J Clin Microbiol 2014; 52:4394-4397. http:// doi.org/10.1128/JCM.02468-14.

33. Leon SR, Ramos LB, Vargas SK, et al. Laboratory Evaluation of a dual-path platform assay for rapid point-of-care HIV and syphilis testing. J Clin Microbiol 2016; 54:492-494. http://doi.org/10.1128/JCM. 03152-15

34. Omoding D, Katawera V, Siedner M, et al. Evaluation of the SD Bioline HIV/Syphilis Duo assay at a rural health center in Southwestern Uganda. BMC Res Notes 2014; 7:746. http://doi.org/10.1186/ 1756-0500-7-746

35. Shimelis T, Tadesse E. The diagnostic performance evaluation of the SD BIOLINE HIV/syphilis Duo rapid test in southern Ethiopia: A cross-sectional study. BMJ Open 2015; 5:e007371. http://doi.org/10. 1136/bmjopen-2014-007371

36. Yin YP, Ngige E, Anyaike C, et al. Laboratory evaluation of three dual rapid diagnostic tests for HIV and syphilis in China and Nigeria. Int $\mathrm{J}$ Gynaecol Obstet 2015; 130:S22-26. http://doi.org/10.1016/j.ijgo. 2015.04.004.

37. Bristow CC, Leon SR, Huang E, et al. Field evaluation of a dual rapid diagnostic test for HIV infection and syphilis in Lima, Peru. Sex Transm Infect 2016; 92:182-185. http://doi.org/10.1136/sextrans-2015-052326.

38. Bristow CC, Severe L, Pape JW, et al. Dual rapid lateral flow immunoassay fingerstick wholeblood testing for syphilis and HIV infections is acceptable and accurate, Port-au-Prince, Haiti. BMC Infect Dis 2016; 16:302. http://doi.org/10.1186/s12879-016-1574-3

39. Bristow CC, Leon SR, Ramos LB, et al. Laboratory evaluation of a dual rapid immunodiagnostic test for HIV and syphilis infection. J Clin Microbiol 2015; 53:311-313. http://doi.org/10.1128/JCM.02763-14.

40. Chesson HW, Heffelfinger JD, Voigt RF, et al. Estimates of primary and secondary syphilis rates in persons with HIV in the United States, 2002. Sex Transm Dis 2005; 32:265-269.

41. Boily M-C, Baggaley RF, Wang L, et al. Heterosexual risk of HIV-1 infection per sexual act: systematic review and meta-analysis of observational studies. Lancet Infect Dis 2009; 9:118-129. http://doi.org/10. 1016/S1473-3099(09)70021-0.

42. Lynn WA, Lightman S. Syphilis and HIV: A dangerous combination. Lancet Infect Dis 2004; 4:456-466. http://doi.org/10.1016/S14733099(04)01061-8.
43. Mwapasa V, Rogerson SJ, Kwiek JJ, et al. Maternal syphilis infection is associated with increased risk of mother-to-child transmission of HIV in Malawi. AIDS 2006; 20:1869-1877. http://doi.org/10.1097/01 .aids 0000244206.41500 .27 .

44. Sanchez J, Lama JR, Peinado J, et al. High HIV and ulcerative sexually transmitted infection incidence estimates among men who have sex with men in Peru: Awaiting for an effective preventive intervention. J Acquir Immune Defic Syndr 2009; 51:S47-51. doi: 10.1097/ QAI.0b013e3181a2671d.

45. Sellati TJ, Wilkinson DA, Sheffield JS, et al. Virulent Treponema pallidum, lipoprotein, and synthetic lipopeptides induce CCR5 on human monocytes and enhance their susceptibility to infection by human immunodeficiency virus type 1. J Infect Dis 2000; 181:283-293. http://doi.org/10.1086/315209.

46. Bristow CC, Larson E, Anderson LJ, et al. Cost-effectiveness of HIV and syphilis antenatal screening: A modelling study. Sex Transm Infect 2016; 92:340-346. doi: 10.1136/sextrans-2015-052367. Epub 2016 Feb 26.

47. Pant Pai N, Kurji J, Singam A, et al. Simultaneous triple point-of-care testing for HIV, syphilis and hepatitis B virus to prevent mother-tochild transmission in India. Int J STD AIDS 2012; 23:319-324. doi: 10.1258/ijsa.2011.011139.

48. Pant Pai N, Daher J. Multiplexed testing for HIV and related bacterial and viral co-infections. Expert Rev Mol Diagn;15:463-469.

49. Nuñez-Forero L, Moyano-Ariza L, Gaitán-Duarte H, et al. Diagnostic accuracy of rapid tests for sexually transmitted infections in symptomatic women. Sex Transm Infect 2016; 92:24-28.doi: 10.1136/sextrans2014-051891. Epub 2015 Jul 1.

50. Gaydos CA, Van Der Pol B, Jett-Goheen M, et al. Performance of the cepheid CT/NG Xpert rapid PCR test for detection of chlamydia trachomatis and Neisseria gonorrhoeae. J Clin Microbiol 2013; 51 : 1666-1672. doi: 10.1128/JCM.03461-12. Epub 2013 Mar 6.

51. Nathan B, Appiah J, Saunders P, et al. Microscopy outperformed in a comparison of five methods for detecting Trichomonas vaginalis in symptomatic women. Int J STD AIDS 2015; 26:251-256. doi: 10.1177/0956462414534833. Epub 2014 May 22

52. Hegazy MM, El-Tantawy NL, Soliman MM, et al. Performance of rapid immunochromatographic assay in the diagnosis of Trichomoniasis vaginalis. Diagn Microbiol Infect Dis 2012; 74:49-53. doi: 10.1016/j.diagmicrobio.2012.05.003. Epub 2012 Jun 21.

53. Badman SG, Causer LM, Guy R, et al. A preliminary evaluation of a new GeneXpert (Gx) molecular point-of-care test for the detection of Trichomonas vaginalis. Sex Transm Infect 2016; 92:350-352. doi: 10.1136/sextrans-2015-052384

54. Samarawickrama A, Cheserem E, Graver M, et al. Pilot study of use of the BioStar Optical ImmunoAssay GC point-of-care test for diagnosing gonorrhoea in men attending a genitourinary medicine clinic. J Med Microbiol 2014; 63:1111-1112. doi: 10.1099/jmm.0.0718520. Epub 2014 Jun 6.

55. Bartelsman M, Straetemans M, Vaughan K, et al. Comparison of two Gram stain point-of-care systems for urogenital gonorrhoea among high-risk patients: Diagnostic accuracy and cost-effectiveness before and after changing the screening algorithm at an STI clinic in Amsterdam. Sex Transm Infect 2014; 90:358-362. doi: 10.1136/ sextrans-2013-051500. Epub 2014 May 23. PMID: 24860102.

56. Khatoon R, Jahan N, Ahmad S, et al. Comparison of four diagnostic techniques for detection of Trichomonas vaginalis infection in females attending tertiary care hospital of North India. Indian J Pathol Microbiol 2015; 58:36-39. doi: 10.4103/0377-4929.151172. PMID: 25673589 .

57. Chiu RYT, Jue E, Yip AT, et al. Simultaneous concentration and detection of biomarkers on paper. Lab Chip 2014; 14:3021-3028. http://doi. org/10.1039/c4lc00532e.

58. Pereira DY, Chiu RY, Zhang SC, et al. Single-step, paper-based concentration and detection of a malaria biomarker. Anal Chim Acta 2015 882:83-89. doi: 10.1016/j.aca.2015.04.040.

59. Chiu RY, Nguyen PT, Wang J, et al. Dextran-coated gold nanoprobes for the concentration and detection of protein biomarkers. Ann Biomed Eng 2014; 42:2322-2332. doi: 10.1007/s10439-014-1043-3.

60. Lin Y-H, Wang S-H, Wu M-H, et al. Integrating solid-state sensor and microfluidic devices for glucose, urea and creatinine detection based 
on enzyme-carrying alginate microbeads. Biosens Bioelectron 2013; 43:328-335. http://doi.org/10.1016/j.bios.2012.12.053.

61. Shin DJ, Zhang Y, Wang T-H. A droplet microfluidic approach to single-stream nucleic acid isolation and mutation detection. Microfluid Nanofluidics 2014; 17:425-430. http://doi.org/10.1007/s10404-0131305-7.

62. Rodriguez NM, Linnes JC, Fan A, et al. Paper-based RNA Extraction, in situ isothermal amplification, and lateral flow detection for low-cost, rapid diagnosis of influenza A (H1N1) from clinical specimens. Anal Chem 2015; 87:7872-7879. doi: 10.1021/acs.analchem.5b01594.

63. Madhivanan P, Krupp K, Hardin J, et al. Simple and inexpensive pointof-care tests improve diagnosis of vaginal infections in resource constrained settings. Trop Med Int Health 2008; 14:703-708. doi: 10.1111/j.1365-3156.2009.02274.x. Epub 2009 Apr 20.

64. Clarke MA, Rodriguez AC, Gage JC, et al. A large, population-based study of age-related associations between vaginal $\mathrm{pH}$ and human papillomavirus infection. BMC Infect Dis 2012; 12:33. doi: 10.1186/1471-2334-12-33.

65. Mathews C, van Rensburg A, Coetzee N. The sensitivity of a syndromic management approach in detecting sexually transmitted diseases in patients at a public health clinic in Cape Town. S Afr Med J 1998; 88:1337-1340.

66. Pant Pai N, Sharma J, Shivkumar S, et al. Supervised and unsupervised self-testing for HIV in high- and low-risk populations: A systematic review. PLoS Med 2013; 10:e1001414. doi: 10.1371/journal. pmed.1001414. Epub 2013 Apr 2.

67. Wingrove I, McOwan A, Nwokolo N, et al. Diagnostics within the clinic to test for gonorrhoea and chlamydia reduces the time to treatment: A service evaluation. Sex Transm Infect 2014; 90:474. doi: 10.1136/sextrans-2014-051580.

68. Wong V, Johnson C, Cowan ER, et al. HIV self-testing in resource limited settings: Regulatory and policy considerations. AIDS Behav 2014; 18:S415-421. doi: 10.1007/s10461-014-0825-9.

69. Pai NP, Wilkinson S, Deli-Houssein R, et al. Barriers to implementation of rapid and point-of-care tests for human immunodeficiency virus infection: Findings from a systematic review (1996-2014). Point Care 2015; 14:81-87.

70. FDA (2011). FDA Guidance Document: Establishing the Performance Characteristics of In Vitro Diagnostic Devices for Chlamydia trachomatis and/or Neisseria gonorrhoea: Screening and Diagnostic Testing. Available at: http://www.fda.gov/RegulatoryInformation/ Guidances/ucm254813.htm.

71. Pai NP, Dhurat R, Potter M, et al. Will a quadruple multiplexed pointof-care screening strategy for HIV-related co-infections be feasible and impact detection of new co-infections in at-risk populations? Results from cross-sectional studies. BMJ Open 2014; 4:e005040. doi: 10.1136/bmjopen-2014-005040.

72. Schachter J. Point-of-care tests using enzyme detection to diagnose Chlamydia trachomatis infection do not work. But when they fail in clinical trials, they reappear under different names. Sex Transm Infect 2016; 92:406-407. doi: 10.1136/sextrans-2015-052508. Epub 2016 Feb 19.

73. Wijesooriya NS, Rochat RW, Kamb ML, et al. Global burden of maternal and congenital syphilis in 2008 and 2012: A health systems modelling study. Lancet Glob Health 2016; 4:e525-533. 